\title{
miR-584 inhibits cell proliferation, migration and invasion in vitro and enhances the sensitivity to cisplatin in human cervical cancer by negatively targeting GLI1
}

\author{
TINGFENG WANG $^{1}$, JUAN FENG ${ }^{1}$ and AIYUN ZHANG ${ }^{2}$ \\ ${ }^{1}$ Department of Gynaecology and Obstetrics, Weifang Maternity and Child Care Hospital; \\ ${ }^{2}$ Department of Gynaecology and Obstetrics, AnQiu Maternity and Child Care Hospital, \\ Weifang, Shandong 261042, P.R. China
}

Received March 18, 2019; Accepted October 29, 2019

DOI: $10.3892 /$ etm.2020.8449

\begin{abstract}
Cervical cancer is the most lethal malignancy amongst women worldwide. MicroRNAs (miRNAs/miRs) play a critical role in the progression of cervical cancer. Compelling evidence indicates that miR-584 acts as a tumor suppressor in some types of cancers. However, the function of miR-584 in cervical cancer has not been illustrated. In the present study, the effects and mechanism of miR-584 in the process of proliferation, migration and invasion, and drug sensitivity to cisplatin in cervical cancer were determined. miR-584 expression decreased markedly in cervical cancer tissues and cell lines compared with healthy control samples. Dual-luciferase reporter assays confirmed that glioma-associated oncogene 1 (GLI1) is a novel molecular target of miR-584. The overexpression of miR-584 inhibited the expression of GLI1, reduced cell proliferation, migration and invasion, and induced apoptosis in HeLa cells. However, the silencing of miR-584 in CaSki cells produced the opposite effects. In addition, the overexpression of GLI1 in HeLa-cells overexpressing miR-584 markedly reversed the miR-584-induced inhibitory effect. Flow cytometry results showed that miR-584 enhanced cisplatin sensitivity by promoting chemotherapy-induced apoptosis. Therefore, miR-584 acted as a tumor suppressor miRNA and might be a novel target gene for future cervical cancer treatments.
\end{abstract}

\section{Introduction}

Cervical cancer is associated with some of the highest rates of morbidities and mortalities in females worldwide (1). Although

Correspondence to: Dr Juan Feng, Department of Gynaecology and Obstetrics, Weifang Maternity and Child Care Hospital, 407 Qingnian Road, Weifang, Shandong 261042, P.R. China

E-mail: smallbee328@163.com

Key words: cervical cancer, microRNA-584, glioma-associated oncogene 1, cisplatin, proliferation, migration and invasion the clinical outcome of therapy in patients improves through early diagnosis, surgical resection, and chemotherapy/radiation therapy, the prognosis remains pessimistic (2). The main reasons for the death of patients with cervical cancer are cancer progression, metastasis, and drug resistance (3). Therefore, gaining full understanding of the molecular mechanisms underlying the tumorigenesis and tumor progression of cervical cancer and developing novel methods of treatment for patients with this disease is essential.

MicroRNAs (miRNAs/miRs) are a class of small ( $\sim 22$ nucleotides) non-coding RNAs that function as negative regulators of gene expression by binding to complimentary sequences mainly in the 3'-untranslated regions (3'-UTRs) of specific mRNAs (4). Accumulating evidence indicates that miRNAs play a critical role in regulating various biological processes, such as cell proliferation, metastasis, and drug resistance $(4,5)$. miR-584 serves as tumor suppressor in several tumor types (6-13). However, the same miRNA may either serve as an oncogene or a tumor suppressor, depending on the characteristic of its target genes (14). Therefore, the biological function and molecular mechanism of miR-584 in cervical cancer must be determined.

Glioma-associated oncogene 1 (GLI1) is an important transcription factor in the Hedgehog signalling pathway that can regulate transcription and expression of various target genes, such as c-MYC and PTCH1, thereby affecting cell proliferation, apoptosis, migration and invasion $(15,16)$. GLI1 acts as an oncogene and is regulated by miR-361-3p in cervical cancer (17). However, to the best of our knowledge, the association between miR-584 and GLI1 has not been illustrated.

In the current study, the expression level of miR-584 was analyzed in cervical cancer to illustrate the biological functions and the molecular mechanism of miR-584.

\section{Materials and methods}

Tissue collection. Cervical cancer tissues and adjacent normal tissues were obtained from 30 patients who underwent surgical resection between December 2015 and December 2017 at the Department of Gynaecology and Obstetrics, Weifang Maternity and Child Care Hospital. Patients were excluded 
from this study if they were receiving any anticancer treatment. Tissues were immediately frozen in liquid nitrogen and stored at $-80^{\circ} \mathrm{C}$ for further usage. The experimental protocol was approved by the Ethics Committee of Weifang Maternity and Child Care Hospital, and a signed informed consent was obtained from all patients before the study.

Cell culture and cell transfection. Human cervical cancer cell lines (C33A, CaSki, HeLa and SiHa) and human immortalized normal cervical cell line Ect1/E6E7 were obtained from American Type Culture Collection. 293T cells were obtained from The Cell Bank of Type Culture Collection of the Chinese Academy of Sciences (Shanghai, China). All cell lines were grown in DMEM (HyClone; GE Healthcare Life Sciences) supplemented with 10\% FBS (HyClone; GE Healthcare Life Sciences), $100 \mathrm{U} / \mathrm{ml}$ penicillin and $100 \mu \mathrm{g} / \mathrm{ml}$ streptomycin (Beyotime Institute of Biotechnology) in a humidified atmosphere at $37^{\circ} \mathrm{C}$ containing $5 \% \mathrm{CO}_{2}$. The miR-584 mimics (mimics) (5'-AGUCAAGGUCCAAUUGGUCCGA-3'), miR-584 mimics negative control (miR-NC) (5'-ACUACU GAGUGACAGUAGA-3'), miR-584 inhibitors (inhibitors) (5'-GCUCUGCUACACUCGGUACUA-3') and miR-584 inhibitors negative control (anti-NC) (5'-UUCUCCGAACGU GUCACGUTT-3') were synthesized by Guangzhou RiboBio, Co., Ltd. Full-length GLI1 from the human cDNA library was cloned into a pcDNA3.1 vector (Invitrogen; Thermo Fisher Scientific, Inc.). The pcDNA3.1 vector alone (empty plasmid) served as a negative control. The HeLa cells were transfected with miR-NC (50 $\mathrm{nM})$ or miR-584 mimics $(50 \mathrm{nM})$ and/or pcDNA3.1/GLI1 vector $(100 \mathrm{nM})$ or pcDNA3.1 $(100 \mathrm{nM})$ using Lipofectamine ${ }^{\circledR} 2000$ reagent (Invitrogen; Thermo Fisher Scientific, Inc.) according to the manufacturer's protocol. The CaSki cells were transfected with anti-NC $(50 \mathrm{nM})$ or miR-584 inhibitors $(50 \mathrm{nM})$ using Lipofectamine ${ }^{\circledR} 2000$ reagent (Invitrogen; Thermo Fisher Scientific, Inc.) according to the manufacturer's protocol. Following transfection for $48 \mathrm{~h}$, the cells were collected for subsequent experiments.

Cell Counting Kit-8 (CCK-8) assays. HeLa and CaSki cell viability was detected using CCK-8 (Beyotime Institute of Biotechnology). HeLa and CaSki cells $\left(1 \times 10^{3}\right.$ cells/well $)$ were cultured in 96-well plates for 0, 24, 48, and $72 \mathrm{~h}$. At the indicated timepoints, $10 \mu \mathrm{l}$ of CCK- 8 was added to each well and incubated for $3 \mathrm{~h}$. The absorbance of each well was then determined using Multiskan MK3 (Thermo Fisher Scientific, Inc.).

Colony formation assay. HeLa and CaSki cells (400 cells/well) were seeded in six-well plates and cultured in DMEM(HyClone; GE Healthcare Life Sciences) supplemented with $10 \%$ FBS (HyClone; GE Healthcare Life Sciences) for 10 days. The cells were then fixed with $1 \mathrm{ml} 4 \%$ paraformaldehyde (PFA; Beyotime Institute of Biotechnology) and stained with crystal violet. The number of colonies containing $>50$ cells was counted with a light microscope (magnification, x100; Olympus Corporation).

Transwell assay. The migration and invasion capability of cells was detected with Corning Transwell chambers (Corning, Inc.). For invasion capability detection, the Transwell membrane filter was precoated with $30 \mu \mathrm{l}$ of Matrigel (BD Biosciences) at $37^{\circ} \mathrm{C}$ for $4 \mathrm{~h}$. In the migration and invasion examination, HeLa and CaSki cells (5x10 $0^{4}$ cells) were resuspended in $100 \mu \mathrm{l}$ DMEM without FBS and transferred to the upper chambers. A total of $600 \mu 1$ of DMEM supplemented with $10 \%$ FBS was added to the lower chamber. Cells were incubated for $12 \mathrm{~h}$ before being fixed with $4 \%$ PFA, stained with $0.5 \%$ crystal violet at room temperature for $20 \mathrm{~min}$, and the stained cells from six random fields were counted, and the images were captured under a light microscope (Olympus Corporation; magnification, $\mathrm{x} 100$ ).

Western blotting. Tissue samples and the treated cells were lysed in radioimmunoprecipitation assay buffer (Beyotime Institute of Biotechnology). Protein concentrations were determined using a BCA Protein Assay kit (Pierce; Thermo Fisher Scientific, Inc.). Equal amounts of protein extracts ( $30 \mu \mathrm{g}$ total protein/lane) were resolved by $10 \%$ SDS-PAGE and transferred onto PVDF membranes, followed by blocking with 5\% nonfat dried milk for $1 \mathrm{~h}$ at room temperature. Subsequently, the PVDF membranes were incubated with primary GLI1 antibody (cat. no. ab49314; 1:500; Abcam) and $\beta$-actin antibody (cat. no. ab8227; 1:1,000; Abcam) overnight at $4^{\circ} \mathrm{C}$ with gentle agitation, and then treated with a Horseradish peroxidase-labeled goat anti-rabbit secondary antibody $(1: 1,000$; cat. no. ab150077; Abcam) for $2 \mathrm{~h}$ at room temperature. $\beta$-actin was used as a loading control. The protein bands were visualized using an enhanced chemiluminescence system (Beyotime Institute of Biotechnology).

$R N A$ extraction and reverse transcription-quantitative PCR (RT-qPCR). Total RNA from cells and tissues was extracted with TRIzol reagent (Thermo Fisher Scientific, Inc.). cDNA was synthesized using a TaqMan ${ }^{\circledR}$ MicroRNA Reverse Transcription kit (Thermo Fisher Scientific, Inc.) according to the manufacturer's instructions. To quantify the miRNA and mRNA, a qPCR assay was performed using $\mathrm{iQ}^{\mathrm{TM}} \mathrm{SYBR}^{\circledR}$ Green Supermix (Bio-Rad Laboratories, Inc.) in an iCycler $\mathrm{iQ}^{\mathrm{TM}}$ qPCR detection system (Bio-Rad Laboratories, Inc.). The relative expression levels of miR-584 and GLI1 were calculated as the inverse $\log$ of $\triangle \Delta \mathrm{Cq}$ and normalized to the reference gene (18). The thermocycling conditions were as follows: $95^{\circ} \mathrm{C}$ for $10 \mathrm{~min}$; followed by 40 cycles of $95^{\circ} \mathrm{C}$ for $15 \mathrm{sec}$ and $60^{\circ} \mathrm{C}$ for $1 \mathrm{~min}$; annealing at $55^{\circ} \mathrm{C}$ for $30 \mathrm{sec}$; and elongation at $72^{\circ} \mathrm{C}$ for $3 \mathrm{~min}$. To analyze GLI1 mRNA expression, $\beta$-actin acted as an internal control. To examine the expression of miR-584, U6 was used as the internal control. The following primer pairs were used: miR-584 forward, 5'-TGCAATGTGTGTGTTAGC CA-3', and reverse, 5'-ATCATTGCTCCTTGGATGGT-3'; GLI1 forward, 5'-TACTCACGCCTCGAAAACCT-3' and reverse, 5'-AGGACCATGCACTGTCTTGA-3'; U6 forward, 5'-TGCGGGTGCTCGCTTCGCAGC-3' and reverse, 5'-CCA GTGCAGGGTCCGAGGT-3'; $\beta$-actin forward, 5'-GATCAT TGCTCCTCCTGAGC-3' and reverse, 5'-ACTCCTGCTTGC TGATCCAC-3'.

Flow cytometry. Transfected HeLa and CaSki cells were treated with $10 \mu \mathrm{M}$ cisplatin (Jiangsu Hansoh Pharmaceutical Co. Ltd.) for $24 \mathrm{~h}$ at $37^{\circ} \mathrm{C}$. The apoptosis rate of cells was then detected using an Annexin V-PI Assay kit (Beyotime Institute 
A

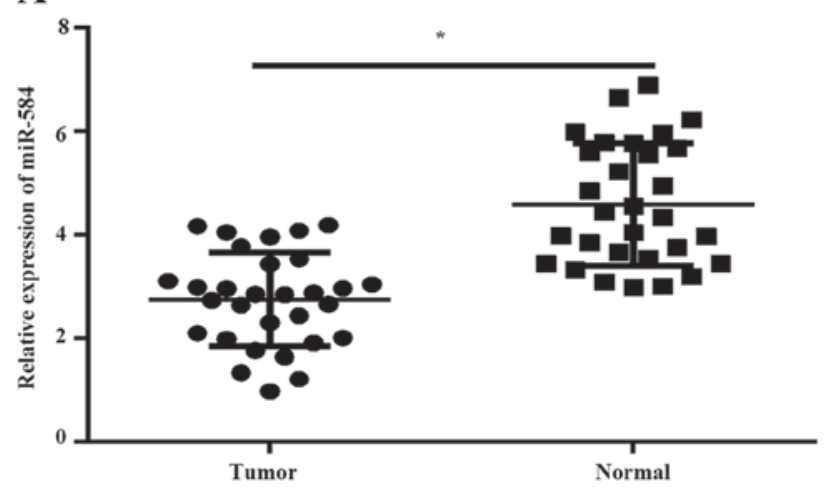

B

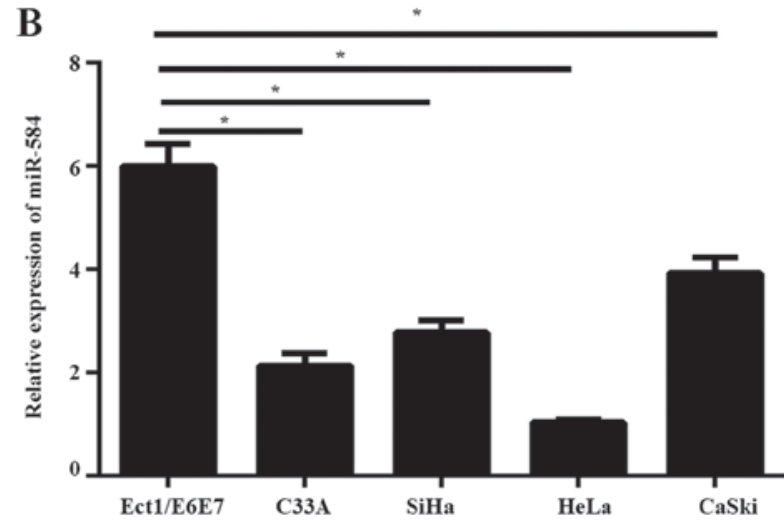

Figure 1. Expression of miR-584 is downregulated in human cervical cancer tissues and cells. (A) RT-qPCR was used to detect the expression of miR-584 in 30 pairs of human cervical cancer tissues and normal tissues. (B) The expression of miR-584 in cervical cancer cell lines and normal cervical cell line Ect1/E6E7 were explored using RT-qPCR. ${ }^{*} \mathrm{P}<0.05$. RT-qPCR, reverse transcription-quantitative PCR; miR, microRNA.

of Biotechnology) according to the manufacturer's instructions. A flow cytometer (Cytomics FC 500 MPL; Beckman Coulter, Inc.) was used to analyze the rate of apoptosis in each sample. Data were analyzed using ModFit LT 3.0 (Verity Software House, Inc.).

Luciferase reporter assay. The wild-type (WT) or mutant (MUT) GLI1-3'UTR, which contained the miR-584 binding sites, was inserted into the psiCHECK2 vector (Promega Corporation) $293 \mathrm{~T}$ cells $\left(1 \times 10^{5}\right.$ cells/well) were co-transfected with $0.1 \mathrm{mg}$ psiCHECK2-WT GLI1-3e miR-5r $0.1 \mathrm{mg}$ psiCHECK2-MUT GLI1-3'-UTR and $10 \mathrm{nM}$ miR-584 mimics or $10 \mathrm{nM}$ miR-584 inhibitors using Lipofectamine ${ }^{\circledR} 2000$ reagent (Invitrogen; Thermo Fisher Scientific, Inc.) according to the manufacturer's protocol. Cells were cultured at $37^{\circ} \mathrm{C}$ for $48 \mathrm{~h}$ and luciferase activities were analyzed using dual-luciferase kit (GeneCopoeia, Inc.) according to the manufacturer's protocol. The activity of firefly luciferase was normalized to the corresponding Renilla luciferase activity.

Bioinformatics prediction. To investigate the possible target genes of miR-584, the online prediction system, TargetScan 7.1 software (http://www.targetscan.org), was used.

Statistical analysis. Results are presented as the mean \pm SEM. Significance was established using the SPSS 13.0 software (SPSS, Inc). Data were analyzed using a Student's t-test or one-way analysis of variance followed by Tukey's Honest Significant Difference test. Pearson's correlation analysis was used to analyze the correlation between miR-584 and GLI1 mRNA expression. $\mathrm{P}<0.05$ was considered to indicate a statistically significant difference.

\section{Results}

Expression of miR-584 is downregulated in human cervical cancer tissues and cells. To explore the role of miR-584 in cervical cancer, miR-584 expression was first detected in 30 pairs of cervical cancer tissues and adjacent normal tissues by RT-qPCR. RT-qPCR results illustrated that the expression of miR-584 was significantly downregulated in tumor tissues compared with normal tissues (Fig. 1A). In addition, the expression levels of miR-584 were analyzed in immortalized normal cervical cell line Ect1/E6E7 and four types of cervical cancer cells (C33A, SiHa, HeL and CaSki) using RT-qPCR. The results showed that the expression of miR-584 in cervical cancer cell lines was significantly reduced compared with Ect1/E6E7 cells (Fig. 1B).

miR-584 inhibits cervical cancer cell proliferation and metastasis. To study the effects of miR-584 in cervical cancer progression, miR-584 overexpression or inhibition assays were performed in HeLa and CaSki cells, which contained the lowest or highest endogenous miR-584 expression levels, respectively. The results of the RT-qPCR assay illustrated that miR-584 expression was significantly increased in HeLa cells and significantly downregulated in CaSki cells when compared with controls (Fig. 2A). The results of the CCK-8 (Fig. 2B) and colony formation assay (Fig. 2C) illustrated that the proliferation of HeLa cells transfected with miR-584 mimics was markedly inhibited compared with the miR-NC group. Conversely, a significant increase in cell proliferation was observed in CaSki cells transfected with miR-584 inhibitors when compared with controls (Fig. 2C and D). Furthermore, the Transwell assay illustrated that the migration and invasion ability of the HeLa cells transfected with miR-584 mimics markedly decreased compared to the miR-NC group, while the silencing of miR-584 increased the migration and the invasion capability of the CaSki cells (Fig. 2E and F).

GLII is a molecular target gene of miR-584. To assess the underlying mechanisms of miR-584 in the progression of cervical cancer cells, Targetscan was used to predict potential target genes of miR-584. GLI1 mRNA 3'-UTR was found to contain highly conserved binding sites for miR-584 (Fig. 3A). A luciferase reporter assay was performed to analyze the association between GLI1 and miR-584. A miR-584 mimic or inhibitor and a luciferase reporter plasmid containing a wt or mut 3'-UTR binding site of human GLI1 were co-transfected into $293 \mathrm{~T}$ cells. miR-584 mimics significantly decreased the luciferase activity in $293 \mathrm{~T}$ cells containing the GLI1 wt 3'-UTR but failed to suppress this activity in cells with the mut GLI1 3'-UTR, while the miR-584 inhibitors enhanced the luciferase activity in $293 \mathrm{~T}$ cells containing the GLI1 wt 
A

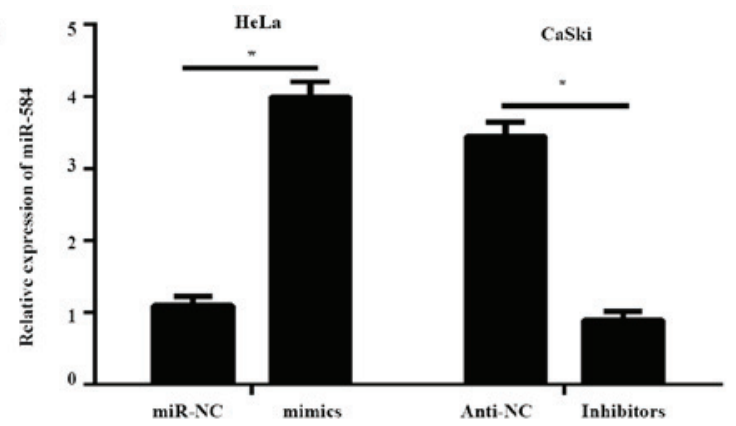

C

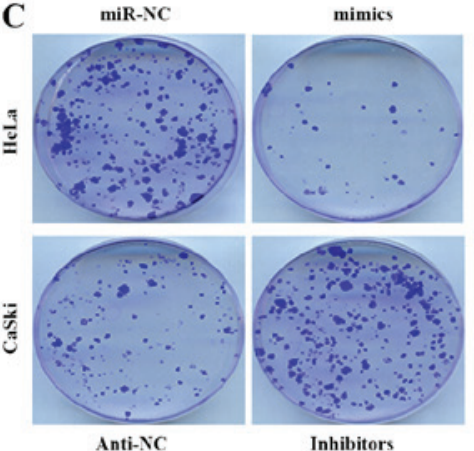

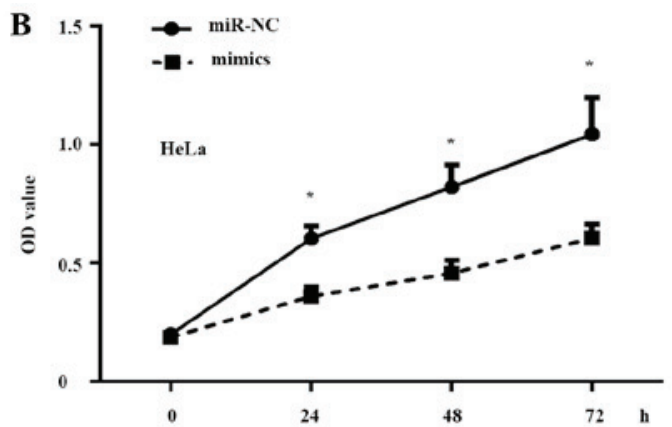

D
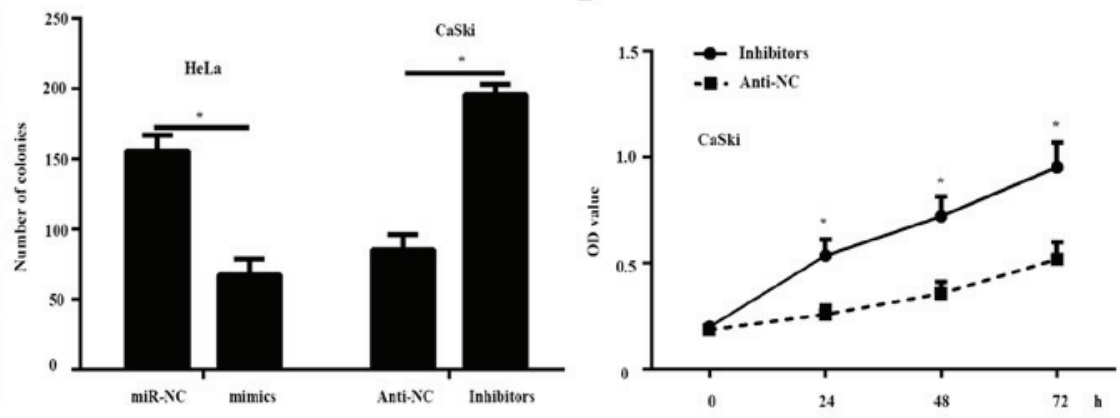

E

HeLa

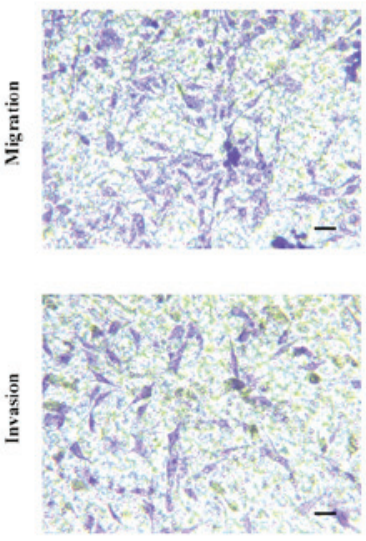

miR-NC

F
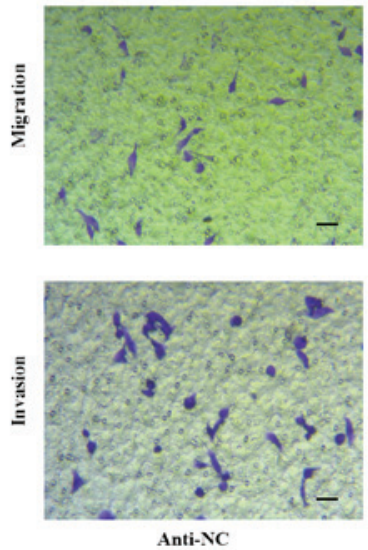
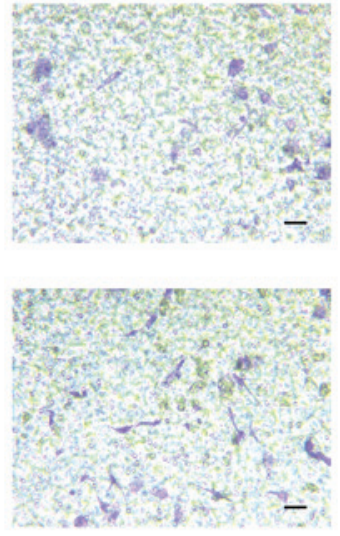

mimics
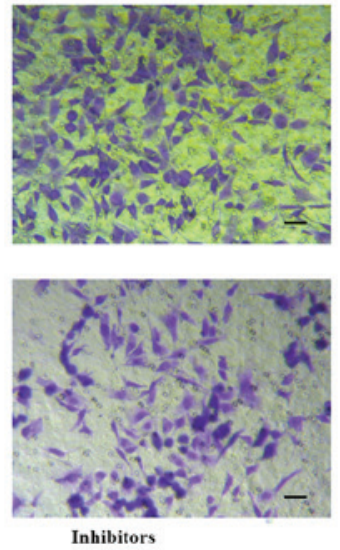
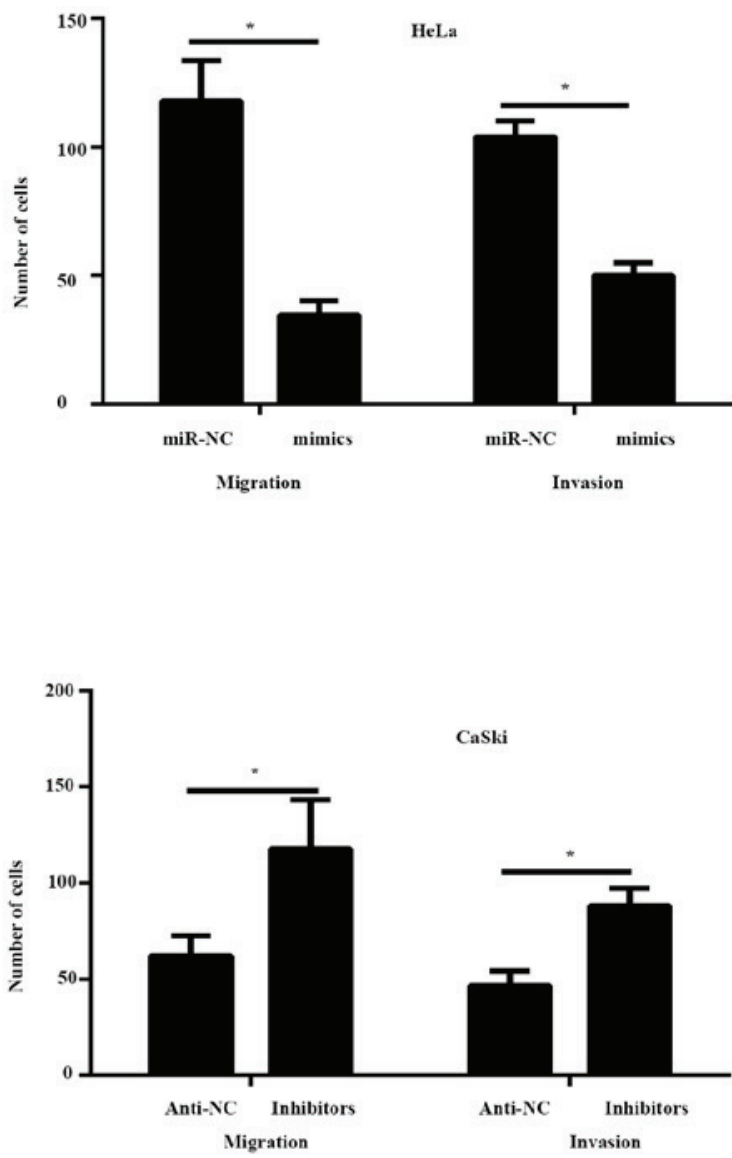

Figure 2. miR-584 inhibits cervical cancer cell proliferation, migration and invasion. (A) miR-584 expression in HeLa cells transfected with mimics or miR-NC and CaSki cells transfected with inhibitors or anti-NC was detected by reverse transcription-quantitative PCR. (B) The cell viability of HeLa cells was tested with a CCK-8 assay. (C) A colony formation assay was used to analyze the proliferation rates of HeLa and CaSki cells. (D) The cell viability of CaSki cells was tested with a CCK-8 assay. (E) A Transwell assay was used to analyze the migration and invasion capability of HeLa cells. (Scale bar, $100 \mu$ m; magnification, $\mathrm{x} 100)$. (F) A Transwell assay was used to analyze the migration and invasion capability of CaSki cells (Scale bar, $100 \mu \mathrm{m}$; magnification, $\mathrm{x} 100)$. $\mathrm{P}<0.05$. miR-NC, mimic negative control; CCK-8, Cell Counting Kit-8; miR, micro-RNA; anti-NC, inhibitor negative control. 
A

GLI1 WT 5' ...UUUCCUGAUAAUAAAgGAACUGC... 3'

, . . . .

miR-584 3' UCGGACCAACCGGACCUUGACU 5'

GLI1 MUT 5' UUUCCUGAUAAUAAAGCUUGAGC... 3'
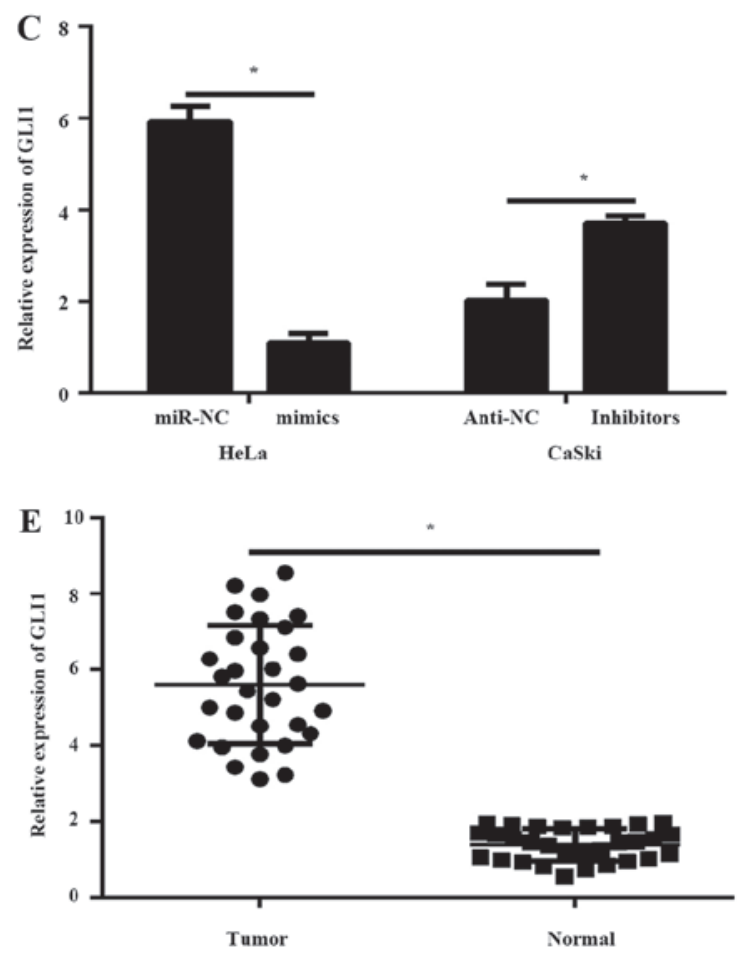
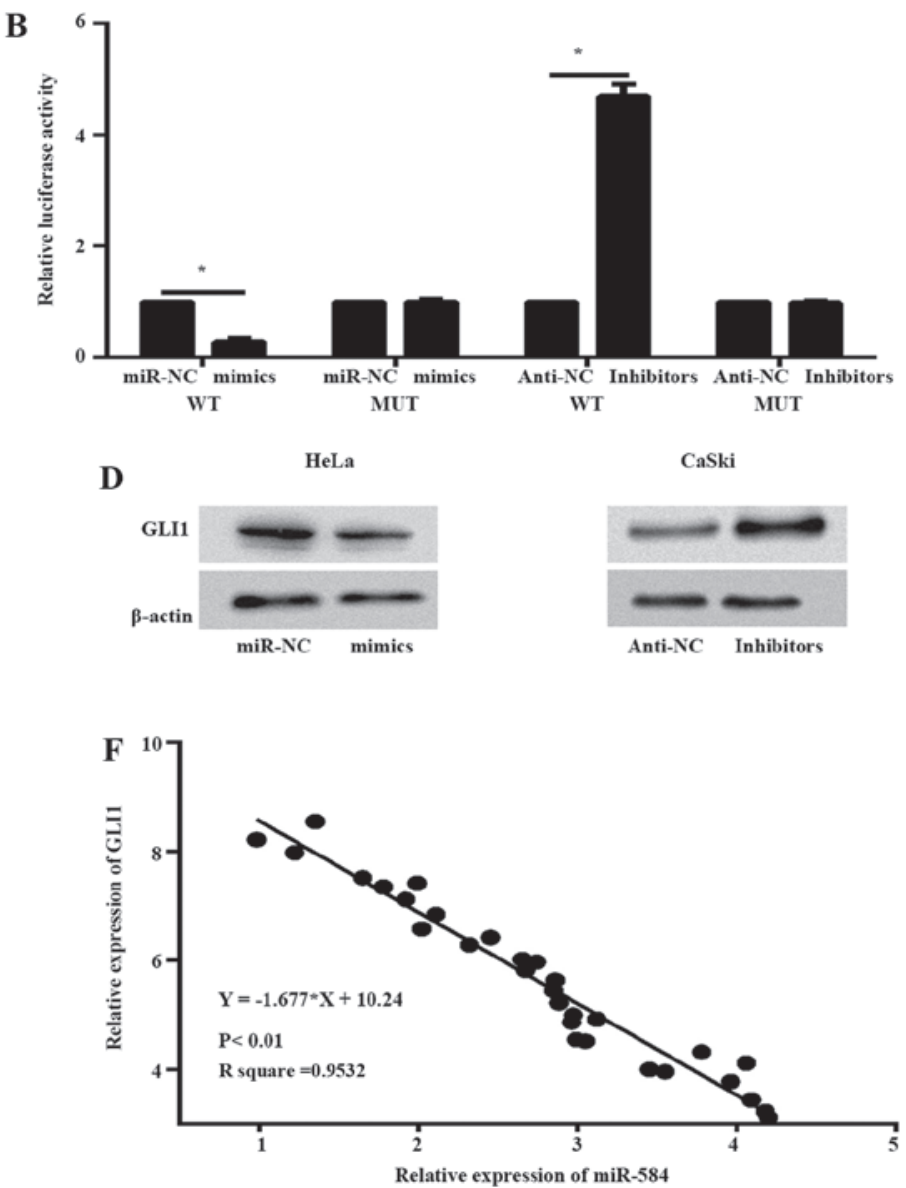

Figure 3. GLI1 is a direct target gene of miR-584. (A) The 3'-UTR of GLI1 mRNA includes a highly conserved binding site for miR-584. (B) 293T cells were co-transfected with miR-584 mimics or inhibitors and wt GLI1 mRNA 3'-UTR or mut GLI1 mRNA 3'-UTR. Luciferase activity was analyzed following 24 h of transfection. (C) mRNA expression of GLI1 was analyzed in HeLa and CaSki cells. $\beta$-actin was used as an internal control. (D) The protein expression levels of GLI1 were detected in HeLa and CaSki cells. $\beta$-actin served as an internal control. (E) Relative GLI1 mRNA expression was analyzed in tumor tissues the corresponding adjacent normal tissues by reverse transcription-quantitative PCR. $\beta$-actin served as internal control. (F) The correlation between the mRNA expression of GLI1 and miR-584 in cervical cancer tissue samples analyzed by Pearson's correlation analysis. 'P<0.05. 3'UTR, 3'-untranslated region; wt, wild-type; mut, mutant; miR, microRNA; miR-NC, mimic negative control; anti-NC, inhibitor negative control; GLI1, glioma-associated oncogene 1.

3'-UTR (Fig. 3B). These data demonstrated that GLI1 is a specific target of miR-584 (Fig. 3B). Furthermore, the results of the RT-qPCR and western blotting illustrated that overexpression of miR-584 downregulated the expression of GLI1 in HeLa cells both at mRNA (Fig. 3C) and protein (Fig. 3D) levels compared with miR-NC cells, while the silencing of miR-584 in CaSki cells resulted in opposite results, further confirming that GLI1 is a target gene of miR-584. The mRNA expression levels of GLI1 were subsequently analyzed in breast cancer samples and corresponding normal tissues using RT-qPCR. The expression of GLI1 at the mRNA level was significantly higher in breast cancer tissues than in adjacent normal tissues (Fig. 3E). In addition, Pearson's correlation analysis (linear regression analysis) showed that the mRNA expression level of GLI1 was inversely correlated with the expression of miR-584 in breast cancer tissues (Fig. 3F).

GLII is a functional target gene of miR-584. To further illustrate whether GLI1 is a function target gene of miR-584, HeLa cells were co-transfected with miR-584 mimic and GLI1 plasmid or empty plasmid. The western blot results illustrated that the protein expression of GLI1 was markedly increased in HeLa cells co-transfected with both miR-584 mimic and GLI1 plasmid compared with cells co-transfected with miR-584 mimic and empty plasmid (Fig. 4A). The CCK-8 (Fig. 4B) and colony formation assay (Fig. 4C) illustrated that the overexpression of GLI1 significantly rescued the proliferation rate decreased by miR-584 mimics. Furthermore, the Transwell assay illustrated that the metastatic capability of HeLa cells co-transfected with both miR-584 mimics and GLI1 plasmid was enhanced compared with HeLa cells co-transfected with miR-584 mimic and empty plasmid (Fig. 4D). These data illustrated that GLI1 was a functional target gene of miR-584.

miR-584 promotes drug sensitivity to cisplatin in cervical cancer cells. The association between miR-584 and cisplatin was determined. Flow cytometry was used to analyze the effects of miR-584 on the drug sensitivity of HeLa and CaSki cells. The results illustrated that overexpression of miR-584 increased the proportion of apoptotic cells compared with the miR-NC group in HeLa and CaSki cells (Fig. 5A and B). In addition, the combination of miR-584 and cisplatin significantly enhanced the apoptosis rate of HeLa and CaSki cells compared with cisplatin or miR-584 mimics, respectively (Fig. 5A and B). These data suggested that miR-584 enhanced the sensitivity of cervical cancer cells to cisplatin. 

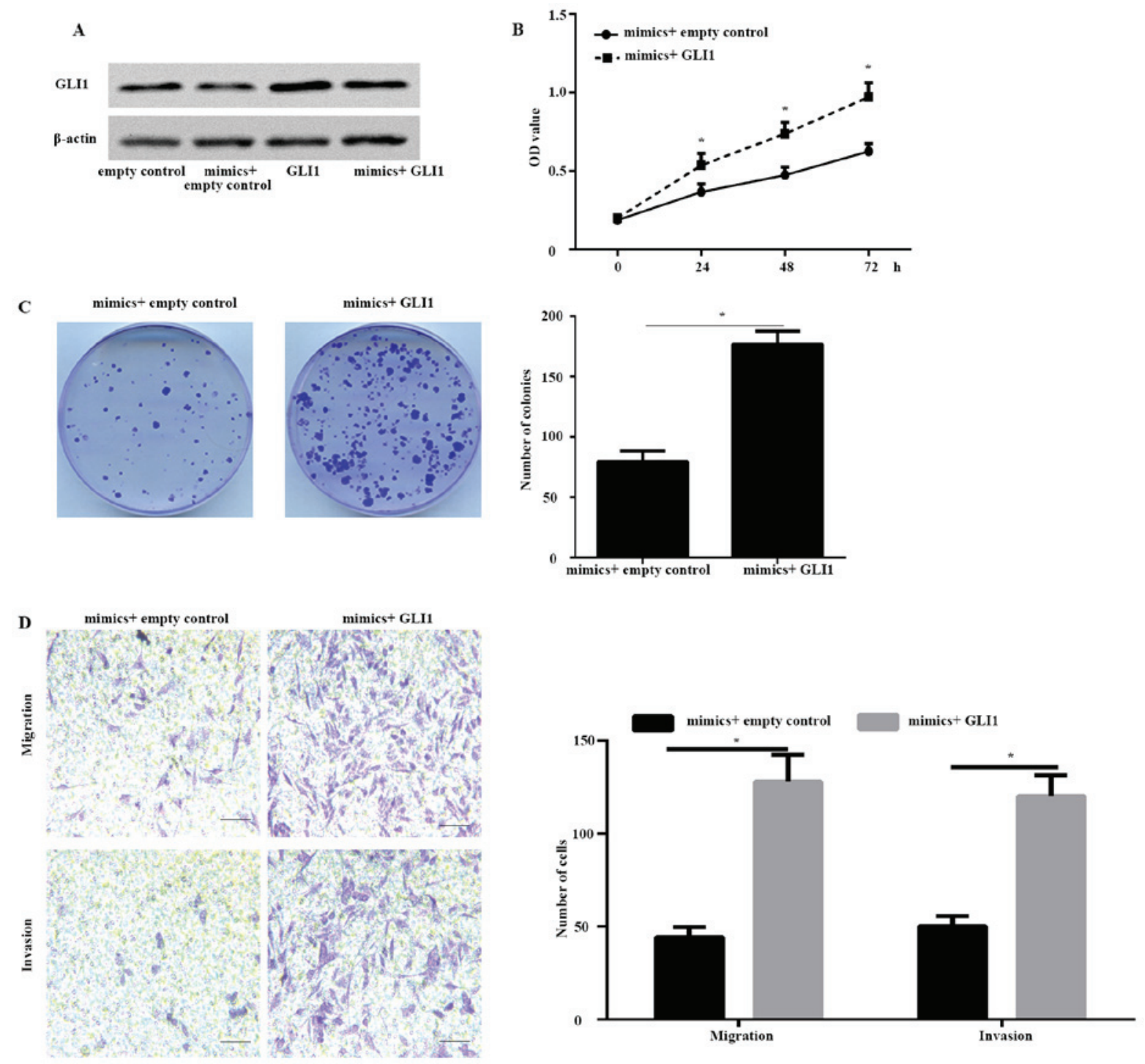

Figure 4. GLI1 is a functional target gene of miR-584. (A) HeLa cells were co-transfected with miR-584 mimics and GLI1 plasmid or empty plasmid. The protein expression levels of GLI1 were then analyzed by western blotting. (B) Cell proliferation of HeLa cells was analyzed using a Cell Counting Kit-8 assay. (C) Cell proliferation of HeLa cells was analyzed using a colony formation assay. (D) A Transwell assay was used to analyze the migration and invasion capability of HeLa cells (Scale bar, $100 \mu \mathrm{m}$; magnification, x100). "P<0.05. miR, microRNA; GLI1, glioma-associated oncogene 1.

\section{Discussion}

A number of studies have illustrated the relationship between cancer progression and deregulated miRNA expression (19). Several miRNAs act as either tumor suppressors or oncogenes involved in cervical cancer progression (19). Decreased expression of tumor suppressor miRNAs leads to enhanced oncogene translation, which in turn enhances tumor development. Similar effects are caused by oncogenic miRNA overexpression, which contributes to the inhibition of tumor suppressor genes (20).

miR-584 acts as a tumor suppressor and is downregulated in some types of cancer (6-13). However, to the best of our knowledge, the expression and functions of miR-584 in cervical cancer have not been elucidated. In the present study, the expression of miR-584 was markedly downregulated in cervical cancer tissues and cell lines compared with adjacent normal cervical tissues and Ect1/E6E7 cells. The current study illustrated the role of miR-584 in cervical cancer cell proliferation, metastasis and apoptosis. The results suggested that miR-584 overexpression inhibited the viability, proliferation, migration and invasion of these cells and enhanced the apoptosis rate in vitro.

Recent studies confirmed that miRNAs inhibit the expression of specific target genes, which leads to tumor occurrence. In medulloblastoma, miR-584 was downregulated in tumor tissues compared with normal tissues, and histone deacytelase 1 and eIF4E3 were the direct target genes of miR-584 (21). In lung cancer, miR-584 was downregulated and inhibited cell growth and metastasis by directly targeting metadherin (6). In gastric cancer, miR-584 directly targeted the matrix metalloproteinase-14 (MMP-14) promoter to repress YY1-facilitated MMP-14 expression and inhibited gastric cancer progression (7). In the current study, miR-584 directly targeted GLI1 and negatively regulated the expression of GLI1 to inhibit cervical cancer progression. GLI proteins, including GLI1, GLI2 and GLI3, are zinc finger transcription factors and are the main effectors of the Hedgehog signalling (22). In 

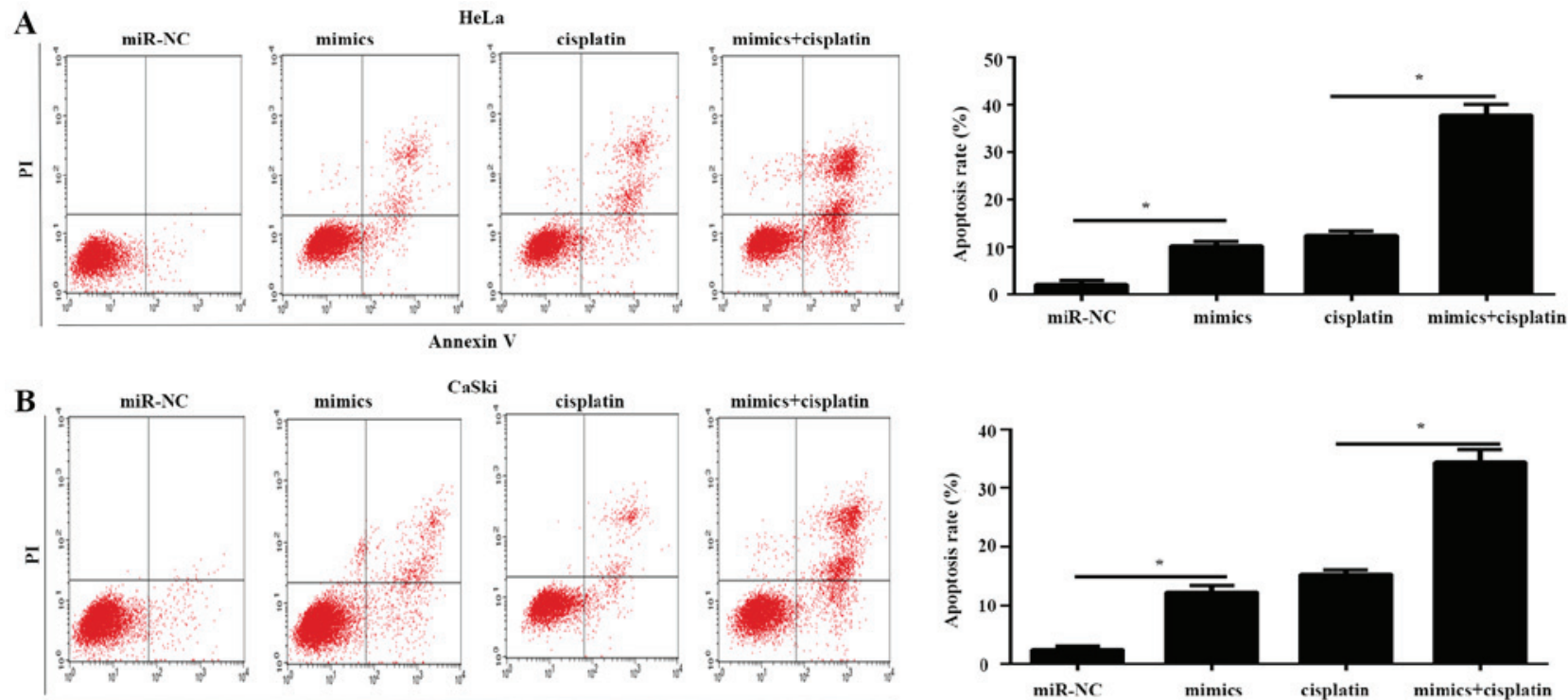

Figure 5. miR-584 promotes drug sensitivity to cisplatin in cervical cancer cells. HeLa and CaSki cells transfected with miR-584 mimics or miR-NC were incubated with or without $10 \mu \mathrm{M}$ cisplatin for $24 \mathrm{~h}$. Overexpression of miR-584 increased the proportion of apoptotic cells compared with the miR-NC group in HeLa and CaSki cells. The combination of miR-584 and cisplatin significantly enhanced the apoptosis rate of HeLa and CaSki cells compared with cisplatin or miR-584 mimics, respectively. (A) The apoptosis rate of HeLa. (B) The apoptosis rate of CaSki. ${ }^{\text {P }}<0.05$. miR, microRNA; miR-NC, mimic negative control.

primary cilium, GLI1 dissociates from the negative regulator suppressor of fused, is converted into its activated form and translocates to the nucleus (22). The translocation of GLI1 enhances the downstream target oncogene expression, such as cyclin D1 and homeobox protein NANOG (22). GLI1 is overexpressed in numerous types of cancer, such as non-small cell lung cancer, gastric cancer, pancreatic cancer and colon cancers, and acts as an oncogene in these tumors $(15,23)$. The current study confirmed that GLI1 is upregulated in cervical cancer tissues, as reported previously (24). miR-584 decreased the expression of GLI1 through the direct binding of the 3'-UTR of GLI1. In addition, the inverse correlation between miR-584 expression and mRNA expression of GLI1 in cervical cancer tissues further supported this conclusion. Moreover, GLI1 overexpression reduced the effects of miR-584 on cell survival and metastasis in cervical cancer. Together, these findings suggested that GLI1 was indeed a direct target gene of miR-584.

Chemoresistance in cancer is the main cause of treatment failure (3). Recent data indicated that aberrant miRNA expression was closely linked to chemoresistance by targeting genes related to chemosensitivity or chemoresistance (25). However, specific chemoresistance-related miRNAs are largely unknown. Cisplatin resistance is a major obstacle to the successful treatment of cervical cancer (3). The current study further illustrated the association between the sensitivity of cells to cisplatin and miR-584 in cervical cancer. miR-584 had a negative impact on cisplatin resistance in HeLa cells. In the current study, it was indicated that GLI1 was a direct target gene of miR-584. Therefore, the underlying mechanism of miR-584 to drug resistance may be through GLI1.

In conclusion, the current study demonstrated that miR-584 is markedly downregulated in human cervical cancer tissues and cell lines. Overexpression of miR-584 inhibited proliferation, invasion, and migration, while enhancing cell apoptosis rates and chemosensitivity to cisplatin in cervical cells. GLI1 was identified as the molecular and biological target gene of miR-584. These data illustrated that miR-584 may serve as a novel therapeutic target in cervical cancer.

\section{Acknowledgements}

Not applicable.

\section{Funding}

No funding was received.

\section{Availability of data and materials}

The datasets used and/or analyzed during the current study are available from the corresponding author on reasonable request.

\section{Authors' contributions}

TW and AZ conceived and designed the experiments. TW, JF and $\mathrm{AZ}$ conducted all of the experiments. TW and JF wrote and revised the manuscript. All authors read and approved the final manuscript.

\section{Ethics approval and consent to participate}

The study was approved by the Ethics Committee of Weifang Maternity and Child Care Hospital. Prior written informed consent was obtained from each patient.

\section{Patient consent for publication}

Not applicable. 


\section{Competing interests}

The authors declare that they have no competing interests.

\section{References}

1. Torre LA, Bray F, Siegel RL, Ferlay J, Lortet-Tieulent J and Jemal A: Global cancer statistics, 2012. CA Cancer J Clin 65 87-108, 2015.

2. Burki TK: Cervical cancer: Screening and risk with age. Lancet Oncol 15: e107, 2014.

3. Waggoner SE: Cervical cancer. Lancet 361: 2217-2225, 2003.

4. Bartel DP: MicroRNAs: Target recognition and regulatory functions. Cell 136: 215-233, 2009.

5. Shen J, Stass SA and Jiang F: MicroRNAs as potential biomarkers in human solid tumors. Cancer Lett 329: 125-136, 2013.

6. Zhang Y, Wang Y and Wang J: MicroRNA-584 inhibits cell proliferation and invasion in non-small cell lung cancer by directly targeting MTDH. Exp Ther Med 15: 2203-2211, 2018.

7. Zheng L, Chen Y, Ye L, Jiao W, Song H, Mei H, Li D, Yang F, Li H, Huang $\mathrm{K}$ and Tong Q: miRNA-584-3p inhibits gastric cancer progression by repressing Yin Yang 1-facilitated MMP-14 expression. Sci Rep 7: 8967, 2017.

8. Li Q, Li Z, Wei S, Wang W, Chen Z, Zhang L, Chen L, Li B, Sun G, $\mathrm{Xu}$ J, et al: Overexpression of miR-584-5p inhibits proliferation and induces apoptosis by targeting WW domain-containing E3 ubiquitin protein ligase 1 in gastric cancer. J Exp Clin Cancer Res 36: 59, 2017.

9. Orlandella FM, Di Maro G, Ugolini C, Basolo F and Salvatore G: TWIST1/miR-584/TUSC2 pathway induces resistance to apoptosis in thyroid cancer cells. Oncotarget 7: 70575-70588, 2016.

10. Xue H, Guo X, Han X, Yan S, Zhang J, Xu S, Li T, Guo X, Zhang P, Gao X, et al: MicroRNA-584-3p, a novel tumor suppressor and prognostic marker, reduces the migration and invasion of human glioma cells by targeting hypoxia-induced ROCK1. Oncotarget 7: 4785-4805, 2016.

11. Xiang J, Wu Y, Li DS, Wang ZY, Shen Q, Sun TQ, Guan Q and Wang YJ: miR-584 suppresses invasion and cell migration of thyroid carcinoma by regulating the target oncogene ROCK1. Oncol Res Treat 38: 436-440, 2015.

12. Wang XP, Deng XL and Li LY: MicroRNA-584 functions as a tumor suppressor and targets PTTG1IP in glioma. Int J Clin Exp Pathol 7: 8573-8582, 2014.
13. Ueno K, Hirata H, Shahryari V, Chen Y, Zaman MS, Singh K, Tabatabai ZL, Hinoda Y and Dahiya R: Tumor suppressor microRNA-584 directly targets oncogene Rock-1 and decreases invasion ability in human clear cell renal cell carcinoma. Br J Cancer 104: 308-315, 2011.

14. Wang L, Wang B, Fang M, Guo F and Cui M: Identification of microRNAs and target genes involved in serous ovarian carcinoma and their influence on survival. Eur J Gynaecol Oncol 35: 655-661, 2014.

15. Didiasova M, Schaefer L and Wygrecka M: Targeting GLI transcription factors in cancer. Molecules 23: pii: E1003, 2018.

16. Mastrangelo $\mathrm{E}$ and Milani M: Role and inhibition of GLI1 protein in cancer. Lung Cancer 9: 35-43, 2018.

17. Zhao D and Cui Z: MicroRNA-361-3p regulates retinoblastoma cell proliferation and stemness by targeting hedgehog signaling. Exp Ther Med 17: 1154-1162, 2019.

18. Livak KJ and Schmittgen TD: Analysis of relative gene expression data using real-time quantitative PCR and the 2(-Delta Delta C(T)) method. Methods 25: 402-408, 2001.

19. Hasanzadeh M, Movahedi M, Rejali M, Maleki F, Moetamani-Ahmadi M, Seifi S, Hosseini Z, Khazaei M, Amerizadeh F, Ferns GA, et al: The potential prognostic and therapeutic application of tissue and circulating microRNAs in cervical cancer. J Cell Physiol 234: 1289-1294, 2019.

20. Shenouda SK and Alahari SK: MicroRNA function in cancer: Oncogene or a tumor suppressor? Cancer Metastasis Rev 28: 369-378, 2009.

21. Abdelfattah N, Rajamanickam S, Panneerdoss S, Timilsina S, Yadav P, Onyeagucha BC, Garcia M, Vadlamudi R, Chen Y, Brenner A, et al: MiR-584-5p potentiates vincristine and radiation response by inducing spindle defects and DNA damage in medulloblastoma. Nat Commun 9: 4541, 2018.

22. Sabol M, Trnski D, Musani V, Ozretic P and Levanat S: Role of GLI transcription factors in pathogenesis and their potential as new therapeutic targets. Int J Mol Sci 19: pii: E2562, 2018.

23. Skoda AM, Simovic D, Karin V, Kardum V, Vranic S and Serman L: The role of the Hedgehog signaling pathway in cancer: A comprehensive review. Bosn J Basic Med Sci 18: 8-20, 2018.

24. Chaudary N, Pintilie M, Hedley D, Fyles AW, Milosevic M, Clarke B, Hill RP and Mackay H: Hedgehog pathway signaling in cervical carcinoma and outcome after chemoradiation. Cancer 118: 3105-3115, 2012.

25. Calıskan M, Güler H and Bozok Cetintas V: Current updates on microRNAs as regulators of chemoresistance. Biomed Pharmacother 95: 1000-1012, 2017.

(i) $\Theta$ This work is licensed under a Creative Commons Attribution-NonCommercial-NoDerivatives 4.0 International (CC BY-NC-ND 4.0) License. 